MATEC Web of Conferences 33, 02008 (2015)

DOI: $10.1051 /$ matecconf/ 20153302008

(c) Owned by the authors, published by EDP Sciences, 2015

\title{
A probabilistic model for martensitic avalanches
}

\author{
John M. Ball ${ }^{1, a}$, Pierluigi Cesana ${ }^{1,2, b}$ and Ben Hambly ${ }^{1, c}$ \\ ${ }^{1}$ Mathematical Institute, University of Oxford, Andrew Wiles Building, Radcliffe Observatory Quarter, Woodstock Road, Oxford \\ OX2 6GG, U.K. \\ ${ }^{2}$ Institute of Mathematics for Industry, Kyushu University - Australia Branch, Department of Mathematics and Statistics, La Trobe \\ University, Bundoora, VIC 3086, Australia
}

\begin{abstract}
We present a probabilistic model for the description of martensitic avalanches. Our approach to the analysis of the model is based on an associated general branching random walk process. Comparisons are reported for numerical and analytical solutions and experimental observations.
\end{abstract}

\section{Introduction}

A martensitic phase-transformation is a first-order diffusionless transition involving a change of shape of the underlying crystal lattice. In a temperature-induced transformation, upon cooling from the high-temperature parent phase (austenite), atoms rapidly rearrange themselves in a coordinated manner, causing a symmetry breaking of the unit cell into a mixture of twin-related variants (martensite). This cooperative reorganization of the atoms results in the formation both of interfaces separating the different martensitic variants, and of habit planes separating the austenite from laminates formed from these variants.

Discontinuous, although kinematically compatible, deformation gradients evolve in space and time, causing the propagation of ultrasonic elastic waves, which generate acoustic emissions (AEs) that are a characteristic signature of martensitic transformations. This process is activated by the free-energy difference between austenite and martensite, and takes place through a sequence of jerks or avalanches. It is thought that the intermittent character of this transformation is strongly influenced by the disorder of the system, that is by lattice defects and impurities, as well as by the onset of long-range interactions of an elastic nature.

In recent years there has been an intensive research effort on the experimental investigation of the jerky character of martensitic transformations, specifically via the statistical analysis of the emitted acoustic signals. An important feature which has been observed in this analysis is that the amplitude, duration and energy of the acoustic emissions exhibit a power-law behaviour characterized by a critical exponent. This is the signature of a system which evolves in the absence of a length-scale or time scale and it is often connected to self-similarity. Experimental evi-

\footnotetext{
ae-mail: ball@maths.ox.ac.uk

be-mail: p.cesana@latrobe.edu.au

ce-mail: hambly@maths.ox.ac.uk
}

dence [8] shows that the exponents determined experimentally for different materials can be grouped into universality classes according to the symmetry of the martensitic phase. This observation seems to suggest that there are features of the dynamics of the transformation that depend only on the symmetry reduction at the transition [8].

In this contribution we present a simple probabilistic model for the description of avalanches, in which we imagine that the martensite forms through the nucleation and propagation of thin martensitic plates parallel to the allowed habit planes. The plates are idealized as planar surfaces which nucleate and propagate according to a stochastic process in time and space. An acoustic emission is assumed to be associated with the formation of each plate. By encoding our probabilistic model into a general branching random walk, we are able to obtain predictions for the asymptotic behaviour of the statistical density of the main features of the random pattern generated. Despite the intrinsic limitations of the model, we show that some of the key features observed in experiments are captured by both our numerical simulations and our analytical predictions.

For a different theoretical approach to martensitic avalanches in terms of a corresponding sandpile model see $[6,7]$.

\section{The model}

We consider a single crystal, or a single grain of a polycrystal, which transforms on cooling from austenite to martensite. As explained in the introduction, we idealize the evolution of the martensitic microstructure in terms of successive nucleation of thin martensitic plates, which propagate in the direction of their corresponding habit planes until they encounter an existing plate.

In the simplest two-dimensional form of the model we consider a unit square and suppose that there are only two possible habit plane directions, which are taken to be horizontal and vertical. In the first step a nucleation point $x$

This is an Open Access article distributed under the terms of the Creative Commons Attribution License 4.0, which permits unrestricted use distribution, and reproduction in any medium, provided the original work is properly cited. 
is chosen uniformly at random in the square. With probability $p$ the vertical direction is chosen and with probability $1-p$ the horizontal direction, where $0<p<1$. A plate forms consisting of the intersection of the straight line through $x$ in the chosen direction with the square. Thus after the first step the square has been divided into two rectangles. In the second step, a choice is made of one of the rectangles, and a new plate is nucleated from a point chosen uniformly at random from this rectangle, again consisting of the intersection of the straight line in either the vertical or horizontal direction through the nucleation point with the rectangle. Equivalently, we can think of the nucleation occuring uniformly at random at a point on one of the sides of the rectangle, with corresponding probabilities. This process is repeated, resulting in a successive division of the square into a family of smaller and smaller rectangles by line segments representing plates, where each new plate extends maximally until it encounters an existing plate or the boundary of the square. A rather similar evolution, with the lines representing needle domains, is seen in the computations of avalanches in [10], using a simple model of a ferroelastic material under shear.

There are different possible choices for how the rectangle for the next nucleation point is chosen. One possibility would be to choose a point uniformly at random from the square, which with probability 1 will lie in the interior of some rectangle. A martensitic microstructure resulting from a 5000-event simulation of this simple symmetric $(p=1 / 2)$ model is shown in Fig. 1. In what follows, un-

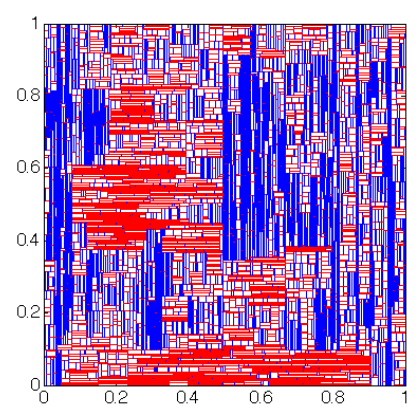

Figure 1. Microstructure for the 2-direction model composed of 5000 randomly generated events.

less otherwise specified, we choose the rectangle with the largest area as that containing the next nucleation point, which will enable us to obtain closed form solutions for the asymptotics of the number of rectangles of different aspect ratios.

In order to describe the model more precisely, we consider each rectangle as determined by the lengths $(a, b)$ of its horizontal and vertical sides respectively. As the nucleation point is uniformly located in the rectangle, and chooses with probability $p$ to form a vertical plate and with probability $1-p$ to form a horizontal one, we can see that the rectangle $(a, b)$ is divided into two smaller rectangles having sides

$$
(a U, b) \text { and }(a(1-U), b) \text { with probability } p
$$

or

$$
(a, b U) \text { and }(a, b(1-U)) \text { with probability } 1-p,
$$

where $U$ is a uniform random variable in $[0,1]$. The case $p=1 / 2$ corresponds to a material in which growth of vertical and horizontal plates is equally probable.

More generally we could consider a single crystal occupying a three-dimensional domain $\Omega$, which we take for simplicity to be convex (that is the closed line segment joining any two points of $\Omega$ lies in $\Omega$ ). Corresponding to a given symmetry of the martensite, there will in general be a finite number of possible habit-plane normals (for example, there are 24 for a cubic-to-tetragonal transformation). The first step in our caricature of the evolution of the martensite now involves nucleating a plate at a random point having one of these normals with prescribed probabilities (e.g. equal probabilities), so that the plane through the nucleation point divides $\Omega$ into two convex parts $\Omega_{1}$ and $\Omega_{2}$. Choosing one of these, say $\Omega_{1}$, for the next randomly chosen nucleation point, together with another randomly chosen normal, the plane through the nucleation point with this normal divides $\Omega_{1}$ into two further convex regions, and so on.

Our two-dimensional model could be viewed as corresponding to a square planar section of $\Omega$, which the possible habit planes intersect in a finite number $n$ of possible straight line directions. Thus a more general twodimensional model would involve choosing one of these directions with prescribed probabilities $p_{i} \geq 0,1 \leq i \leq n$, with $\sum_{i=1}^{n} p_{i}=1$. Again we require that new nucleating lines propagate until they reach the boundary of the square or subdomain in which they nucleate. The square is thus rapidly split into a mixture of up to $2 n$-sided convex polygons, which results in a very rich and complicated pattern (for $n=3,4$ see Figs 2 and 3).

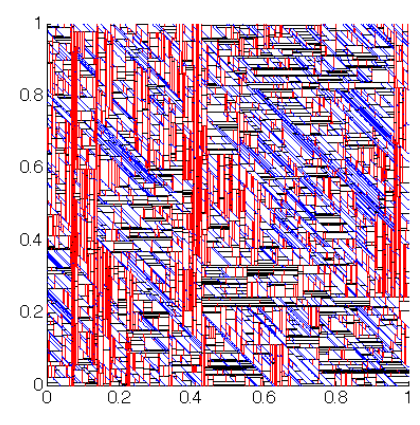

Figure 2. Microstructure for the 3-direction model composed of 5000 randomly generated events, the three directions being horizontal, vertical and at $\mathrm{a}-45^{\circ}$ angle with respect to the horizontal.

We refer the interested reader to an upcoming paper by the group of A. Planes and E. Vives for an alternative approach to the modelling of martensitic avalanches in a discrete version of this model [9]. 


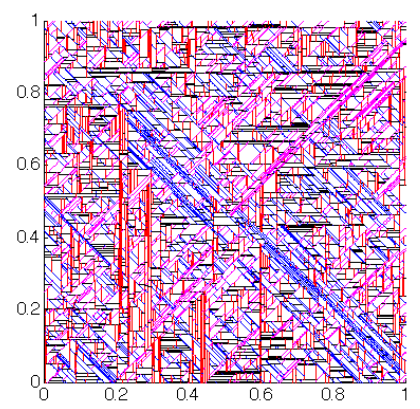

Figure 3. Microstructure for the 4-direction model composed of 5000 randomly generated events, the four directions being horizontal, vertical and at $\pm 45^{\circ}$ to the horizontal.

\section{Analysis via a general branching random walk}

The model can be transformed into a general branching random walk (GBRW), a stochastic process describing systems of particles that move and branch over the course of their lifetimes. An individual particle in the GBRW has a life-length and a reproduction point process giving the birth times and positions of its offspring. These are chosen from a probability distribution and each offspring of the individual then evolves independently. In order to map our model into a GBRW we regard each rectangle of side-lengths $(a, b)$ as a particle in the positive quadrant $\mathbb{R}_{+}^{2}=\{(x, y): x \geq 0, y \geq 0\}$ at a position $(x, y)=(-\log a,-\log b)$. We then introduce an artificial time parameter which will enable us to encode the area of rectangles and is unrelated to any time scale in the formation of martensite. The process starts at time $t=0$ with an initial particle at the origin $(0,0)$ representing the unit square. The mechanism for the evolution of the GBRW is that a particle at $(x, y)$, born at time $\sigma$, then reproduces independently of other particles and has two children at positions $(x-\log U, y)$ at time $\sigma-\log U$ and $(x-\log (1-U), y)$ at time $\sigma-\log (1-U)$ with probability $p$, or at $(x, y-\log U)$ at time $\sigma-\log U$ and $(x, y-\log (1-U))$ at time $\sigma-\log (1-U)$ with probability $1-p$, where $U$ is a random variable with the uniform distribution on $[0,1]$. The particle then dies at the birth time of its second child. This branching mechanism ensures that, as the area of a rectangle is the product of the uniform random variables used to divide it up, the area of a rectangle corresponding to a particle with birth time $\sigma$ is $e^{-\sigma}$ and hence at time $t$ the offspring of the individuals alive correspond to all rectangles with area less than $e^{-t}$ whose parent rectangles had area greater than $e^{-t}$. The realization of the microstructures from which the histograms shown in Fig. 5 are derived corresponds to a GBRW as above with $p=1 / 2$ run until time $t \approx 6.9$.

A classical branching process will either become extinct or grow exponentially. In the case of the general branching random walk there is a shape theorem which indicates the region, after rescaling, in which the number of individuals will grow at an exponential rate (see Biggins $[2,3])$. The growth rates in this region describe the pro- portion of rescaled rectangles of different aspect ratios that persist in the limit. Thus we can recover the asymptotics of small rectangles from this GBRW limit theorem.

For our simple 2-direction model for martensitic plates the asymptotic shape can be computed explicitly and will be presented in a forthcoming paper [4]. By skipping all the technicalities as well as the precise mathematical formulation of the problem, here we report the essential result of the shape theorem for the asymptotic density of rectangles of a certain size.

Let $A$ be a closed convex set with non-empty interior in $\mathbb{R}_{+}^{2}$ which overlaps with the domain of finiteness of $f$, the Legendre transform of the cumulant generating function for the reproduction process. Let $N_{t}(A)$ denote the number of particles of the GBRW in $A$ at time $t$. The asymptotic shape theorem of [2] gives, in the sense of almost sure convergence, that under some weak conditions on $f$

$$
\lim _{t \rightarrow \infty} \frac{\log N_{t}(t A)}{t}=\sup _{(x, y) \in A} f(x, y)
$$

when the supremum is strictly positive. If the supremum is negative, then the limit is 0 . In the setting of our simple model it is possible to calculate the function $f$ explicitly. The theorem cannot be applied directly, as the domain of finiteness of $f$ has no interior, making it a boundary case of the asymptotic shape theorem, but nevertheless the limit theorem still holds and we state the result:

Theorem. For our simple model we have, in the sense of almost sure convergence, that for A a closed convex subset of $\mathbb{R}_{+}^{2}$ having non-empty interior,

$$
\lim _{t \rightarrow \infty} \frac{\log N_{t}(t A)}{t}=\sup _{(x, y) \in A} f(x, y)
$$

when $\sup _{(x, y) \in A} f(x, y)>0$, where for $0 \leq x \leq 1$,

$$
f(x, y)=\left\{\begin{array}{cc}
\sqrt{1-(2 p-1)^{2}} \sqrt{1-(x-y)^{2}} & \\
+(1-2 p)(x-y) & x+y=1, \\
-\infty & x+y \neq 1 .
\end{array}\right.
$$

We now wish to interpret this asymptotic result for the growth of the number of particles in the GBRW in terms of the growth of the number of rectangles in our model. The theorem shows that the number of particles grows exponentially on closed convex sets which move at linear speed away from the origin, provided the set contains a point on the line $x+y=1$. Loosely speaking, if we take a fixed set and large $t$, we can think of this result as saying that the number of particles in this set will be exponentially large (in $t$ ) if the set contains points on the line $x+y=t$.

Let $C_{x, y} \subset \mathbb{R}_{+}^{2}$ be any closed convex set with nonempty interior containing the point $(x, y)$ and such that $x^{\prime} \geq x, y^{\prime} \geq y$ for all $\left(x^{\prime}, y^{\prime}\right) \in C_{x, y}$. If we take $C_{x, y}$ to be such a set with $x+y=1$, the supremum over $\left(x^{\prime}, y^{\prime}\right) \in C_{x, y}$ in our theorem will be achieved at the point $x, y$. Thus for large $t$ the number of particles $N_{t}\left(t C_{x, 1-x}\right)$ in $t C_{x, 1-x}=\left\{\left(t x^{\prime}, t y^{\prime}\right):\left(x^{\prime}, y^{\prime}\right) \in C_{x, 1-x}\right\}$ is approximately given by $\exp (t f(x, 1-x))$. Alternatively, if we take such a set $C_{x, t-x}$, that is with $x, t-x$ as lowest left point for 
any $0 \leq x \leq t$, then $N_{t}\left(C_{x, t-x}\right)$ is approximately given by $\exp (t f(x / t, 1-x / t))$. That is

$\log N_{t}\left(C_{x, t-x}\right) \approx 4 \sqrt{p(1-p)} \sqrt{x(t-x)}+(1-2 p)(2 x-t)$

We now rewrite this result to describe the number of rectangles $\tilde{N}_{t}\left(S_{a, b}\right)$ that lie in a set $S_{a, b}$, which is closed convex with non-empty interior in the interior of the positive quadrant that lies below and to the left of the point $(a, b) \in[0,1]^{2}$. The function $f(x, y)$ can be rewritten as $\tilde{f}(a, b)$ in terms of the variables $(a, b)$ determining the rectangle sides in $[0,1]^{2}$

$\tilde{f}(a, b)=\left\{\begin{array}{rr}2 \sqrt{p(1-p)} \sqrt{1-(-\log a+\log b)^{2}} & \\ +\infty & a b=e^{-1} \\ -\infty & a b \neq e^{-1}\end{array}\right.$

Thus for large $t$ the number of rectangles grows exponentially on the curve $a b=e^{-t}$. A reinterpretation of (1) is then, for a sufficiently large $t$ and $e^{-t} \leq a \leq 1$,

$$
\begin{array}{r}
\log \tilde{N}_{t}\left(S_{a, e^{-t} / a}\right) \approx 4 \sqrt{p(1-p)} \sqrt{-(\log a)(t+\log a)} \\
-(1-2 p)(2 \log a+t)
\end{array}
$$

and in the symmetric case

$$
\log \tilde{N}_{t}\left(S_{a, e^{-t} / a}\right) \approx 2 \sqrt{-(\log a)(t+\log a)} .
$$

In the following section we will present the results of simulations where we consider the logarithmic version (1), in which we have rescaled the spatial co-ordinates by the elapsed time $t$ so that the logarithm of the asymptotic growth rate function for the number of rectangles in the symmetric case is given by $2 t \sqrt{x(1-x)}$ for the rescaled coordinate $0 \leq x \leq 1$.

The lengths of the martensitic plates are determined by the rectangles in that, in our model, the length of the plate formed when a rectangle is subdivided will be the length of one of the sides of the rectangle in which it forms. Thus the number of plates of a certain length is a function of the GBRW found by considering each particle in the GBRW, and taking as the plate length the length of the side that is not split. If we assume that the acoustic emission from the formation of the plate is proportional to its length we see that the asymptotic result in the theorem can be used to capture the number of acoustic emissions due to small plates. Focussing on the symmetric case, let $N_{t}^{P}(a, b)$, with $e^{-t} \leq a<b \leq 1$, be the number of plates whose length lies in the interval $(a, b)$ at time $t$ when the area of the rectangles is approximately $e^{-t}$. Let $\epsilon>0$ be fixed and small and consider large $t$. Then, for $e^{-t / 2} \leq a \leq 1-\epsilon$, we have $\log N_{t}^{P}(a, a+\epsilon) \approx 2 \sqrt{-(\log a)(t+\log a)}$ and for $e^{-t}+\epsilon \leq$ $a \leq e^{-t / 2}$ we have $\log N_{t}^{P}(a, a-\epsilon) \approx 2 \sqrt{-(\log a)(t+\log a)}$.

In what follows we present and discuss some results obtained from the numerical implementation of the model for avalanches and compare its results with the analytic result coming from this general branching random walk model.

\section{Results and discussion}

Microstructures have been generated according to the 2dimensional version of the stochastic model presented in Section 2 and implemented in Matlab. Fig. 1 corresponds to the 2-direction version of the model in which an interface propagates either in the vertical or horizontal direction. The 3-direction microstructure of Fig. 3 corresponds to a pattern generated by vertical and horizontal directions and a third direction which forms a $-45^{\circ}$ angle with respect to the horizontal direction. By considering the case of $+45^{\circ}$ angles as well we obtain the 4-direction pattern of Fig. 3. All the above examples correspond to mixtures of equiprobable states, i.e. each direction is chosen with a probability $p_{i}=1 / n$, with $i=1, \ldots, n$ and $n$ equal to either 2, 3 or 4 respectively. Practically, the choice of the direction of the interface is implemented by a $n$-valued flag whose status is assumed with uniform probability. Post-processing investigation of Matlab's (pseudo)random number generator shows very small bias in the choice of the directions, in good agreement with the assumption of a mixture of equiprobable variants.

We have generated logarithmic histograms for the expected density of the length $s$ of randomly generated interfaces in a 500-event simulation of a 2, 3 or 4-direction version of the model. From the theorem we do not expect a pure power-law behaviour for the probability densities for $p=1 / 2$. However, the curves plotted in Fig. 4 are shallow and can be easily approximated by an affine function if we neglect the contributions for $s \approx 1$ as well as those for $s \ll 0.1$ (these last ones are not reported in Figs 4 and 5 either).

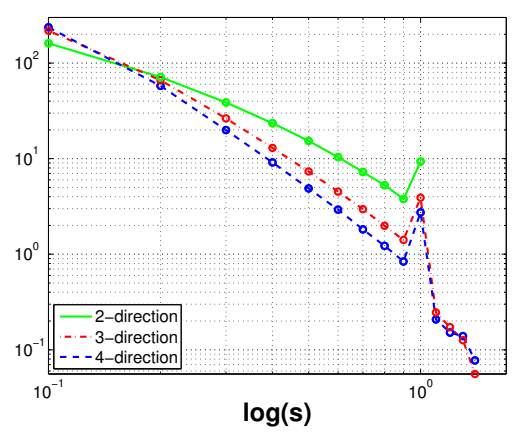

Figure 4. Histograms of the probability density of the length $s$ of the interfaces in a 500-event microstructure. Here averages are obtained over approximately $10^{4}$ realizations of the model.

On a technical note, remember that the shape of a histogram may depend on the number and size of the intervals. Here the choice of the intervals for the graphs of Figs 4 and 5 is considered sufficient in order to stabilize the profile of the density functions resulting from an average of several thousand realizations of the model.

The peak observed in all the histograms for $\log s=0$ correspond to a concentration of interfaces of length $s=1$ across the unit square. This phenomenon can be regarded as a boundary effect and it would not appear in the limit case of an infinitely large domain. 


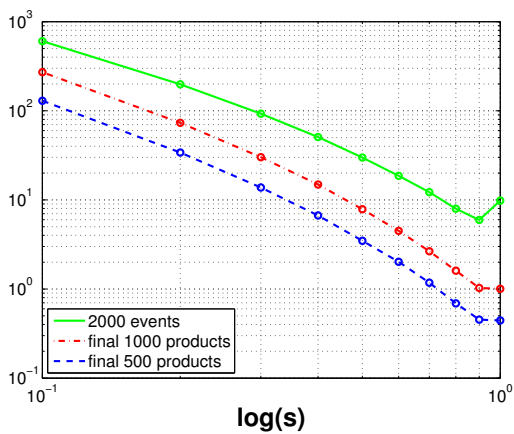

Figure 5. Histograms of the probability density of the length $s$ of interfaces for a 2000-event 2-direction model and evidence of self-similarity. Here and in the following figures averages are obtained over approximately 3000 realizations of the model.

These findings are compared with exponents determined experimentally by the Lab of A. Planes from AE measurements reported in Table 1 [8]. Although it is difficult to relate in a precise and consistent manner information on the geometry of the microstructure (length of interfaces and area of rectangles) with the physical quantities (energy and amplitude of AEs) actually measured, some essential features of the experiment are captured by theory. In particular, the slope of each curve depends on the symmetry of the transformations and the absolute value of the exponent is increasing as the number of variants (and hence the number of habit-plane directions) increases (see Table 1). Predictions coming from our 2-dimensional model provide qualitative information in agreement with the experimental findings. We would expect to obtain better quantitative information on the actual magnitude of the exponent using a full 3-dimensional random nucleation model.

Table 1. Universality class critical exponents $\alpha$ and $\epsilon$ and variant-multiplicity for systems transforming from cubic to selected martensitic symmetries [2]. Here $\alpha$ denotes the exponent related to the probability density function of the amplitude of AE signals while $\epsilon$ denotes the exponent associated with the energy of AE signals.

\begin{tabular}{|c|c|c|c|}
\hline M-symmetry & $\alpha$ & $\epsilon$ & Multiplicity \\
\hline Monoclinic & $3.0 \pm 0.2$ & $2.0 \pm 0.2$ & 12 \\
Orthorhombic & $2.4 \pm 0.1$ & - & 6 \\
Tetragonal & $2.0 \pm 0.3$ & $1.6 \pm 0.1$ & 3 \\
\hline
\end{tabular}

The plots of Figs 1-4 correspond to microstructures obtained by choosing at each time a point uniformly at random from the unit square, while Figs 5-7 were obtained by splitting at each time the existing rectangle of largest area. Although these two approaches seem to be in sufficiently good agreement (as shown by a comparison of corresponding histograms for the lengths of interfaces not reported here) only in the latter case are analytical predictions available and presented in Section 3.

The model has been tested as regards self-similarity of the patterns. From inspection of Figs 1-3 it appears that the microstructure exhibits self-similar features at different scales. This behaviour is well captured by the histogram plots of Fig. 5 where we report the averaged density function for the lengths of the interfaces generated in the 2direction model, corresponding to a time approximately equal to 6.9 (2000 events). This histogram is compared to the averaged density of the lengths of the last 1000 interfaces generated in the same process and to that of the last 500 events only (respectively, dash-dotted and dashed curves), showing that the structure as a whole - as well as that of later generations only - exhibit a similar behaviour.

The theorem provides us with an analytical approximation for the probability density of the rectangles obtained by splitting the microstructure up to a given time $t$. This is represented in the space of normalized coordinates $(x, y)$. The butterfly shaped cloud of Fig. 6 is the result of approximately 3000 simulations, each resulting in the creation of a 2-variant random microstructure. Each point in Fig. 6 corresponds to a rectangle of sides $(a, b)$ with $0 \leq a, b \leq 1$ in the space $(x, y)$ after the log-transformation and the cloud thus obtained can be regarded as a phase diagram for the expected density of rectangles of various sizes. Note that rectangles are concentrated along the line of equation $x+y=1$ or, equivalently, along the curve of the equation $a b=e^{-t}$. This is in agreement with the assumptions of a model for which the largest portion of untransformed material at time $t$ has area $e^{-t}$.

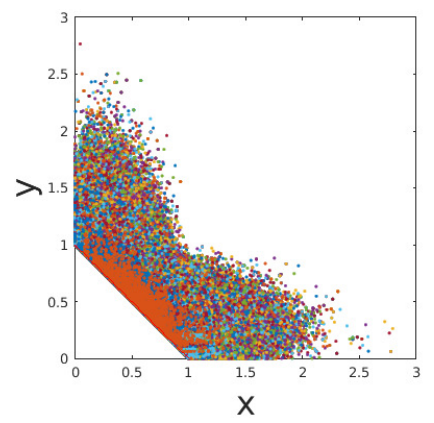

Figure 6. Phase-diagram of the distribution of rectangles in the $(x, y)$ space for the 2-direction model with 2000 events.

The averaged density of the areas of the rectangles is plotted in the form of a 3-dimensional logarithmic histogram in Fig. 7. Thanks to our theorem, an asymptotically exact formula $f(x, y)$ for the averaged density is available and is compared to the histogram. Here some attention is required as the formula $f(x, y)$ only holds in the limit as $t \rightarrow+\infty$, while the histogram plotted in Fig. 7 is obtained from a series of realizations of 2000-event patterns corresponding to $t \approx 6.9$. Recall that $f(x, y)$ is finite-valued on the line $x+y=1$, where for $p=1 / 2$ we have

$$
f(x, 1-x)=2 \sqrt{x(1-x)},
$$

for $0 \leq x \leq 1$. We note that rescaled rectangles tend to concentrate toward the line $x+y=1$ as time increases. Consequently, if we keep the shape and size of the slots fixed, the maximum value of the histogram is an increas- 


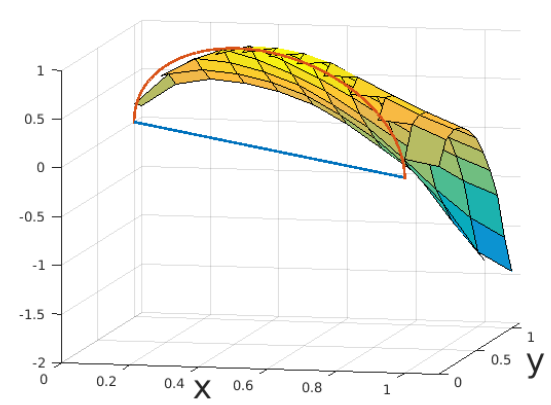

Figure 7. 3-dimensional logarithmic histogram plot for the probability distribution of rectangles in the $(\mathrm{x}, \mathrm{y})$ space for the 2direction model with 2000 events, and comparison with analytical solution $f(x, y)$ on the line $x+y=1$.

ing function of time. In Fig. 7 we choose to normalize the histogram so that its maximum corresponds to that of $f(x, 1-x)$. In this manner, although some quantitative information on the histogram is lost, we are able to see the agreement between the shape of the analytic solution and that of the normalized density of rectangles even for a relatively small finite time $t$.

\subsection{Summary and conclusion}

We have presented a probabilistic model to describe martensitic transformations occurring as a sequence of avalanches. Our approach is based on branching random walk processes and it is designed to reproduce selfsimilarity of the generated patterns in time and space. Comparisons are reported for the prediction of power-laws for the probability density of some fine features generated in an $n$-variant mixture for numerically simulated mi- crostructure, analytical solutions and experiments, showing a qualitative good agreement.

\section{Acknowledgements}

The research of JMB and PC was supported by the ERC under the EU's Seventh Framework Programme (FP7/2007-2013) / ERC grant agreement no 291053 and that of JMB also by a Royal Society Wolfson Research Merit Award. The authors are grateful to Eduard Vives for several discussions as well as for bringing part of his unpublished work [9] to our attention. This work was written when PC held an ERC funded postdoctoral position at the Mathematical Institute of the University of Oxford.

\section{References}

[1] K. Bhattacharya, Microstructure of Martensite (Oxford University Press, 2003)

[2] J. D. Biggins, in Classical and modern branching random processes (IMA Vol. Math. Appl. 84, Springer, New York, 1997) pp 19-39

[3] J.D. Biggins, Ann. Appl. Prob., 5, 1008-1024 (1995)

[4] P. Cesana and B. Hambly, in preparation

[5] O. Nerman and Z. Wahrsch. Verw. Gebiete 57, 365-395, (1981).

[6] F.-J. Pérez-Reche, L. Truskinovsky and G. Zanzotto, Phys. Rev. Letters 99, 075501 (2007)

[7] F.-J. Pérez-Reche, L. Truskinovsky and G. Zanzotto, Continuum Mech. Thermodyn. 21, 17-26 (2009)

[8] A. Planes, L. Manosa and E. Vives, J. Alloys and Compounds 577S, S699-S704 (2013)

[9] A. Planes and E. Vives, in preparation

[10] E. K. H. Salje, X. Ding, Z. Zhao, T. Lookman and A. Saxena, Phys. Rev. B 83, 104109 (2011) 